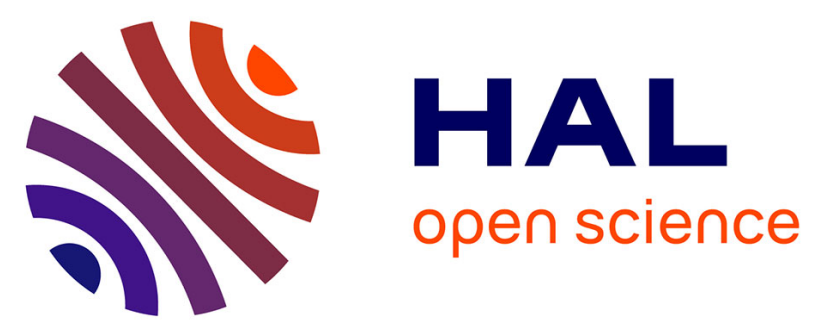

\title{
Circulating microparticles released during dyslipidemia may exert deleterious effects on blood vessels and endothelial function
}

Mohamed E.F. Ousmaal, Abderahim Gaceb, M'Hammed A Khene, Lynda Ainouz, Jean Giaimis, Ramaroson Andriantsitohaina, M. Carmen Martínez, Ahsene Baz

\section{To cite this version:}

Mohamed E.F. Ousmaal, Abderahim Gaceb, M'Hammed A Khene, Lynda Ainouz, Jean Giaimis, et al.. Circulating microparticles released during dyslipidemia may exert deleterious effects on blood vessels and endothelial function. Journal of Diabetes and its Complications, 2020, 34 (10), pp.107683. 10.1016/j.jdiacomp.2020.107683 . hal-03404492

\section{HAL Id: hal-03404492 https://hal.science/hal-03404492}

Submitted on 26 Oct 2021

HAL is a multi-disciplinary open access archive for the deposit and dissemination of scientific research documents, whether they are published or not. The documents may come from teaching and research institutions in France or abroad, or from public or private research centers.
L'archive ouverte pluridisciplinaire $\mathbf{H A L}$, est destinée au dépôt et à la diffusion de documents scientifiques de niveau recherche, publiés ou non, émanant des établissements d'enseignement et de recherche français ou étrangers, des laboratoires publics ou privés. 


\section{Circulating microparticles released during dyslipidemia possess deleterious effects on blood vessels and endothelial function}

Mohamed El Fadel Ousmaal ${ }^{a, b, *}$, Abderahim Gaceb ${ }^{c}$, M’hammed Amine Khene $^{b}$, Lynda Ainouz $^{\mathrm{b}}$, Jean Giaimis ${ }^{\mathrm{d}}$, Ramaroson Andriantsitohaina ${ }^{\mathrm{e}}, \mathrm{M}$. Carmen Martínez ${ }^{\mathrm{e}}$, Ahsene Baz ${ }^{\mathrm{b}}$

${ }^{a}$ Laboratory of valorisation and bio-engineering of natural resources, University of Algiers1, Algiers, Algeria

${ }^{\mathrm{b}}$ Laboratory of Biology and Animal Physiology, ENS Kouba, Algiers, Algeria

${ }^{c}$ Translational Neurology group, Department of Clinical Science, Wallenberg Neuroscience Center, Lund University, Lund, Sweden

d UMR Qualisud- Faculty of Pharmacy, University of Montpellier I, Montpellier, France

e SOPAM, U1063, INSERM, UNIV Angers, SFR ICAT, Bat IRIS IBS, Rue des Capucins, 49100 Angers, France

Mohamed El Fadel Ousmaal, ousmaal_med@hotmail.fr

Abderahim Gaceb, fond.bleu@hotmail.fr

M’hammed Amine Khene, amine.khene@gmail.com

Lynda Ainouz, ainouzlynda@gmail.com

Jean Giaimis, jean.giaimis@univ-montp1.fr

Ramaroson Andriantsitohaina, ramaroson.andriantsitohaina@univ-angers.fr

M. Carmen Martínez, carmen.martinez@univ-angers.fr

Ahsene Baz, baznacim@hotmail.com

Conflict of Interest: The authors have no conflicts of interest to declare.

* Corresponding author at: Mohamed El Fadel Ousmaal

Department of Nature and Life Sciences, Faculty of Science, University of Algiers1, Algiers, Algeria

Tel: +213552574247 .

E-mail address: ousmaal med@hotmail.fr 


\section{Abbreviations:}

AV, Annexin V;

cav-1, caveolin-1;

EC, endothelial cell;

eNOS, endothelial nitric oxide synthase;

HDL-C, high-density lipoprotein cholesterol;

HED, high energy diet;

HUVECs, human umbilical vein endothelial cells;

ICAM-1, intercellular adhesion molecule 1:

$\mathrm{IR}$, insulin receptor;

LDL-C, low-density lipoprotein cholesterol;

MetS, Metabolic syndrome;

MPs, microparticles;

ND, normal diet;

NO, nitric oxide;

P. obesus, Psammomys obesus;

ROS, reactive oxygen species;

TG, triglycerides. 


\section{ABSTRACT}

Circulating microparticles (MPs) are small membrane vesicles that are released from the plasma membrane of circulating blood cells and cells of the vascular wall during activation or death cell. These MPs establish with the vascular cells, especially the endothelial cells, a multiple and complex language. Depending on the type of physiological or pathophysiological signal inducing their release, MPs which are charged with several bioactive molecules may have a vasculoprotective or vasculoaggresive action. This study was designed to compare the effects of circulating MPs from dyslipidemic Psammomys obesus ( $P$. obesus) fed a high-energy diet (HED) with those released from healthy P. obesus fed a normal diet (ND) on ex vivo vascular reactivity, and in vitro ICAM-1, caveolin-1 (cav-1) and eNOS expression and reactive oxygen species (ROS) production on human umbilical vein endothelial cells (HUVECs) for 24 hours. After 12 weeks of feeding with either the HED or ND, circulating MPs from peripheral blood were isolated by differential centrifugation. Both MPS ND $_{\text {and }}$ MPSHED significantly decreased eNOS expression in HUVECS and induced a significant reduction of the maximal of relaxation induced by acetylcholine. Contrary to MPs released from healthy $P$. obesus, MPs from dyslipidemic group significantly decreased cav-1 and eNOS expression and increased ROS production and ICAM-1 expression.

The results of our experimental study suggest that circulating MPs from dyslipidemic $P$. obesus exert a dual impact on the development of atherosclerosis by an antiatherogenic action by reducing the endothelial expression of cav-1, and a proatherogenic action by increasing the endothelial expression of ICAM-1 and endothelial ROS production, and decreasing eNOS expression associated with impaired endothelium-dependent vascular relaxation.

Keywords: Dyslipidemia, microparticles, high-energy diet, ICAM-1, caveolin-1, Psammomys obesus.

\section{Introduction}


Metabolic syndrome (MetS) as one of the leading cause of death worldwide is defined as a set of metabolic disorders and is characterized by a constellation of asymptomatic physiological and biochemical abnormalities. These disorders are vastly related to many cardiovascular diseases including atherosclerosis, stroke, and coronary artery disease [1].

The dysmetabolic state in dyslipidemia, one of the components of MetS, is either associated with an elevation of plasma cholesterol and/or triglycerides (TG), which induces the circulating cells activation and endothelial cell (EC) dysfunction [2], and constitutes the main risk factor for the development of atherosclerotic pathology and cardiovascular morbidity. This disorder can, directly, alter EC functions by ox-LDL-induced inflammation through NF-KB pathway activation [3] and oxidative stress by pro-oxidant enzymes up-regulation [4] or indirectly via circulating cells [5] and their released microparticles (MPs) [6].

MPs are vesicles derived from cell membranes in response to an activating stimulus or apoptosis. In addition to their ability to promote and support the coagulation process, they are known to be involved in many physiological and physiopathological conditions, including immune response, regulation of EC functions, MetS and atherosclerosis [7]. Recent studies have provided evidence for the concept of MPs as true vectors for the intercellular exchange of biological information. MPs can transfer part of their components and their contents to selected target cells, leading to cell activation, phenotypic modification and cell regulation [8]. This role is supported by two major features that allow MPs to be protagonists of the local and systemic communication network. First, the circulating character of MPs in the vascular system that allows MPs to act as shuttles and consequently carry cell signaling transducers in their local environment but, also at a distance from their original site via the presentation of bioactive molecules associated to their membranes [9].

Second, MPs can directly transfer some of their content or components including, proteins, bioactive lipids or RNA to the recipient cells in order to reprogram cell function. This transfer can be sufficiently facilitated by transient membrane interactions, integration of the membrane or the definitive incorporation of MPs into the cell $[9,10]$.

In view of its location to contact with blood, the endothelium is one of the crucial barriers of the body and the primary targets of circulating MPs. Therefore, the endothelium is the seat of several responses to MP subpopulations which differ, according to the disorder, in numbers, composition and function. Acute or prolonged, endothelial responses to MPs can be represented by cellular transformation and functional regulation of the vascular wall [8]. The 
MPs may show proinflammatory properties that lead to endothelial dysfunction and dyslipidemia related cardiovascular complications [11]. It has been widely accepted that MPs of patients with MetS may contribute to endothelial dysfunction, inflammation and vascular hyporeactivity. Indeed, the results obtained by Agouni et al. suggest that MPs from MetS patients leads to impaired endothelium-dependent relaxation in the aorta by decreasing eNOS expression and induces an ex vivo vascular hyporeactivity by increasing both ROS and NO release $[6,7]$.

In the last few years, there has been a growing interest in the mechanism of action of MPs on the vascular wall and particularly, the possibility of using them as a potential therapeutic target in the treatment of MetS related to cardiovascular complications [8]. However, most previous studies did not take into account that MetS is a stimulus-rich environment for the cells, which can be a potential factor for modifying the MP bioactivity. These various stimuli, including obesity, hyperglycemia, dyslipidemia and hypertension, can cause the release of MPs carrying different bioactive components in response to these stimuli and therefore makes more difficult the understanding of the action of each disorder on the modification of MP release and its bioactivity [9].

To elucidate the mechanisms underlying the action of MPs, we present an original approach which MP release was induced by a single disorder; lipid disorder and we used $P$. obesus, a model widely used to study diet-induced obesity as it develops features observed in human MetS. The aim of the study was to investigate the pattern of circulating MPs from dyslipidemic $P$. obesus after feeding HED in comparison with ND-feed $P$. obesus and trying to answer the following question: Is the action of MP release during dyslipidemia a vasculoprotective or vasculoaggresive action? Collectively, this work suggests that the dyslipidemic environment may influence the bioactivity of MPs which may affect vascular homeostasis.

\section{Materials and Methods}

\subsection{Animals and diets}

Twelve male P.obesus weighing 150-200 g (2-3 months) were captured in the Algerian Sahara, more precisely in Biskra, a continental arid area (35 $15^{\prime}$ and $33^{\circ} 30^{\prime} \mathrm{N} ; 4^{\circ} 15^{\prime}$ and $\left.6^{\circ} 45^{\prime} \mathrm{E}\right)$. The animals were maintained under normal diet containing natural plants of the Chenopodiaceae family in a temperature-controlled room $\left(22-25^{\circ} \mathrm{C}\right)$ on a $12-\mathrm{h}$ light-dark cycle. Eight weeks after arrival, the animals were divided into two groups ( $n=6$ per group) and were fed either a high- 
energy diet (HED) or received continuous feeding of a normal diet (ND) for up to 12 weeks, as previously described [12]. On the caloric basis, the HED consisted of $62 \%$ carbohydrate, $27 \%$ protein and $11 \%$ fat, (total $3.9 \mathrm{kcal} / \mathrm{g}$ ), whereas ND contained $50 \%$ carbohydrate, $39 \%$ protein and $11 \%$ fat (total $0.51 \mathrm{kcal} / \mathrm{g}$ ). During the experiment, body weight was measured, and blood samples were taken from the intraorbital retrobulbar plexus from fasted anesthetized P.obesus and were collected in heparin-coated tubes. At the end of the experiment, blood samples from ND and HED group were taken after overnight fasting and were collected either in heparin-coated tubes for biochemical measurements or in tubes containing trisodium citrate (Vacutainers, Becton Dickinson, Le Pont de Claix, France) for MP quantification, to minimize platelet activation, after a cardiac puncture. The animals were used and treated in accordance with the institutional guidelines for animal care, and the protocol was approved by the local ethics committee.

\subsection{Biochemical measurements}

Serum fasting, HDL-C, triglycerides, and glucose levels were prepared by centrifugation at $700 \mathrm{~g}$ for 15 minutes and measured by enzymatic colorimetric methods (BioSystems S.A., Barcelona, Spain), following the manufacturer's protocols. Fasting insulin was measured with a radioimmunoassay kit (Diagnostic Products Corporation, Los Angeles, CA, USA). The lowdensity lipoprotein cholesterol (LDL-C) fraction was estimated using the Friedewald formula $(\mathrm{LDL}-\mathrm{C}=$ total cholesterol $-(0.45 \times \mathrm{TG})+\mathrm{HDL}-\mathrm{C}$, expressed in $\mathrm{mmol} / \mathrm{L})$.

\subsection{MP isolation}

Samples were centrifuged for 15 minutes at $1,500 \mathrm{~g}$ to obtain platelet-rich plasma (PRP). PRP was centrifuged at $13,000 \mathrm{~g}$ for 2 minutes at $4^{\circ} \mathrm{C}$ to obtain platelet-poor plasma (PPP). PPP was immediately frozen in liquid nitrogen and stored at $-80{ }^{\circ} \mathrm{C}$ until MP quantification. As previously described [12], remaining PPP was subjected to two series of centrifugations at $17,000 \mathrm{~g}$ for 45 minutes to pellet MPs. Finally, the MP pellet was resuspended in $200 \mu \mathrm{l}$ of $0.9 \%$ saline salt solution and stored at $4^{\circ} \mathrm{C}$. For in vitro and ex vivo studies, MPs were used at the circulating level detected by flow cytometry in each group (ND and HED). Indeed, flowcount beads were added to each sample (volume/volume) to calculate the MP absolute value; this mixture was then analyzed in a flow cytometer 500 MPL system (Beckman Coulter, Villepinte, France). 
As previously shown [12], means values of circulating MPs were $6917 \pm 2538 \mathrm{MPs} / \mu \mathrm{L}$ of plasma for ND group and $9159 \pm 2491 \mathrm{MPs} / \mu \mathrm{L}$ of plasma for HED group. Phenotypical characterization of the MP subtypes showed a significant increase of MPs from monocyte/neutrophil (CD11b+), procoagulant MPs expressing phosphatidylserine (Annexin $\mathrm{V}+$ ) and a decrease in the endothelial MPs (eMPs) levels in the HED group compared to the ND group.

\subsection{Vascular reactivity}

Thoracic aortas were dissected from the mice immediately after sacrifice. Fat and surrounding tissue were carefully removed under a dissecting microscope. The thoracic aortas were cut into 3-mm length rings, and then incubated for $24 \mathrm{~h}$ with Dulbecco's modified eagle's medium (DMEM, Lonza, Walkersville, USA) supplemented with $10 \%$ fetal bovine serum (FBS, Lonza[CM1]), $0.5 \%$ penicillin/ streptomycin in the absence or presence of MPs, of HED or ND group at their circulating concentrations detected in the blood of animals. Supernatants corresponding to the last MP washing medium have been used as control. Following the incubation, the rings were mounted in a myograph (Danish Myo Technology, Aarhus, Denmark) containing warmed $\left(37{ }^{\circ} \mathrm{C}\right)$, oxygenated $\left(95 \% \mathrm{O}_{2} / 5 \% \mathrm{CO}_{2}\right)$ physiological salt solution of the following composition (mM): $\mathrm{NaCl} 130, \mathrm{KCl} 3.7, \mathrm{KH}_{2} \mathrm{PO}_{4} 1.2, \mathrm{MgSO}_{4} \cdot 7 \mathrm{H}_{2} \mathrm{O} 1.2$, $\mathrm{CaCl}_{2} 1.6$ and glucose 11 . The preparations were equilibrated for at least 60 min under a passive tension of $5 \mathrm{mN}$. After the equilibration period, the response to $\mathrm{KCl}(80 \mathrm{mM})$ containing thromboxane A2 agonist (U-46619, Merck Chemicals Ltd, Nottingham, UK) was assessed in mouse aortas in order to test their maximal contractile capacity. Vasorelaxation responses to cumulative concentrations of acetylcholine (ACh, $1 \mathrm{nM}$ to $10 \mu \mathrm{M}$, Sigma-Aldrich, St. Quentin, Fallavier, France) were examined in vessels precontracted by U-46619 at a concentration induced approximately $80 \%$ maximal contraction. Results were expressed as a percentage of relaxation of thromboxaneA2-induced precontraction, $100 \%$ relaxation was considered when the active tone returned to the baseline level.

\subsection{Cell culture}

HUVECs were isolated by collagenase digestion from freshly delivered umbilical cord veins from normal pregnancies, after patient's informed consent according to the principles expressed in the Declaration of Helsinki. 
HUVECs were cultured in $75 \mathrm{~cm}^{2}$ flasks with RPMI 1640 supplemented with $10 \%$ of fetal bovine serum (FBS, Gibco ${ }^{\circledR}$ ) and antibiotics $(50 \mathrm{U} / \mathrm{ml}$ penicillin, $50 \mu \mathrm{g} / \mathrm{ml}$ streptomycin, $0.1 \mathrm{mg} / \mathrm{ml}$ neomycin. Polfa Tarchomin, Poland) at $37^{\circ} \mathrm{C}$ in a $5 \%$ CO2-95\% air environment.

Upon reaching $70 \%$ confluence, HUVECs were seeded on glass $\mu$-slide (Ibidi, Munich, Germany) to a concentration of $10^{4}$ cells per well. After $48 \mathrm{~h}$, cells were treated for $24 \mathrm{~h}$ with MPs of HED or ND group (at their corresponding circulating level detected in the plasma). Cells were subjected to immunofluorescence staining with cav-1, ICAM-1 and eNOS, ROS detection and F-actin staining. HUVECs were passaged by using $0.25 \%$ trypsin, and passages $4-5$ were used in all experiments. All of the experiments were performed in quadruple.

\subsection{Immunofluorescence staining}

Cells were fixed with $4 \%$ paraformaldehyde and permeabilized with $0.1 \%$ Triton-X100 for 3 min, then incubated overnight $\left(4^{\circ} \mathrm{C}\right)$ with the primary antibodies against cav-1 $(1: 100, \mathrm{BD}$ Biosciences), ICAM-1 (1:100, Santa Cruz[CM2]) or eNOS (1:100, BD Biosciences) and followed, after washing three times, by incubation with their corresponding secondary antibodies (1:100, Molecular Probes, Eugene, OR) for $90 \mathrm{~min}$ at room temperature. After washing, DAPI was applied to stain the nuclei.

\subsection{Detection of reactive oxygen species}

In situ ROS production was evaluated using the oxidative fluorescent dye dihydroethidium (DHE, Sigma-Aldrich). Briefly, HUVECs were washed with PBS three times for 5 min per wash and subsequently incubated with $\operatorname{DHE}(10 \mu \mathrm{M})$ for $30 \mathrm{~min}$ at $37^{\circ} \mathrm{C}$ in a dark humidified chamber. After washing the cells three times with PBS, the fluorescence intensity was detected.

\subsection{F-actin staining}

To stain actin stress fibers, HUVECs were fixed in $2 \%$ paraformaldehyde solution for 20 min and permeabilized with $0.1 \%$ Triton X-100 for $3 \mathrm{~min}$. They were then incubated with phalloidin (50 mg/ml, Sigma-Aldrich) for $90 \mathrm{~min}$ at room temperature in the dark to stain F-actin. After washing with PBS, nuclei were stained with DAPI and washed again.

\subsection{Confocal microscopy and image analysis}

All images were captured using $x 40$ objective of confocal microscopy (CLMS 700, Zeiss, ZEN software). In general, 5 to 6 images of cells were captured randomly. Fluorescence was detected at an excitation and emission wavelength of 488 and $575 \mathrm{~nm}$ for DHE, and 488 and 
$525 \mathrm{~nm}$ for phalloidin. After images adjustment with Zeiss Zen software, fluorescence intensity analysis was performed using a computer-based quantitative color image analysis system in ImageJ (National Institutes of Health; Bethesda, MD) to evaluate the optical density in arbitrary units

\subsection{Data analysis and statistics}

In cumulative concentration-response curves of myograph experiments, differences between values were calculated using non-linear regression analysis. In other experiments, comparisons between two groups were made with non-parametric Mann-Whitney tests. All statistical analysis was performed using GraphPad Prism 5 software (GraphPad Software, Inc., USA). All values in the figures and text are expressed as mean \pm standard error of the mean (SEM) of $n$ observations, where $n$ represents the number of animals studied. A $P$ value less than 0.05 was considered to be statistically significant.

\section{Results}

\subsection{HED induces dyslipidemia without hyperglycemia in $P$. obesus.}

The effects of diets on the levels of serum lipid profile, fat weight and body weight gain were shown in Table 1. After 12 weeks, when compared to the ND, the HED exerted a distinctly pronounced hyperlipidemic effect marked by an increase in total cholesterol, LDL-C and TG. However, no significant differences in HDL-C, glucose and insulin levels were observed between the HED- and ND-fed groups. Moreover, the HED increased visceral fat, subcutaneous fat and body weight gain at 4 weeks and at 12 weeks compared to ND in P.obesus. The obtained data demonstrated that HED developed dyslipidemia without hyperglycemia in P.obesus.

\section{2. $M P S_{N D}$ and $M P S_{H E D}$ impaired ex vivo vessel relaxation induced by $A C h$}

We investigated whether ND and HED affected MPs bioactivity related to vessel relaxation with the aim of understanding the physiological basis behind their involvement in the regulation of vasocontractility.

Treatment with either MPS $\mathrm{S}_{\mathrm{ND}}$ or MPS $\mathrm{S}_{\mathrm{HED}}$ induced a significant reduction of the maximal of relaxation induced by acetylcholine which traduced impairment on endothelial function (Fig. 1A). Interestingly, the reduction of acetylcholine-induced relaxation evoked by MPSHED was not 
different than the effect induced by MPSND (Fig. 1B). These results indicated that both MPSHED and MPSND exhibited a significant effect on vascular homeostasis related to vessel relaxation.

\subsection{Differential effect of MPS $S_{N D}$ and MPS $S_{H E D}$ on eNOS expression and oxidative stress on HUVECs}

Previous studies have suggested that MPs from MetS induced an ex vivo vascular dysfunction by increasing both ROS and NO release [5]. In this study, we investigated whether exposure of HUVECs to MPs of HED group (circulating level detected in the plasma) for $24 \mathrm{~h}$ affected eNOS expression and ROS production, as compared with the MPs of ND group. Our results showed a significant difference between the effect of $M P S_{N D}$ and that of MPS $S_{H E D}$ on the eNOS

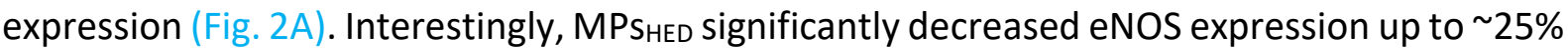
and increased ROS production up to $\sim 75 \%$ on HUVECs (Fig. 2B). These results showed that

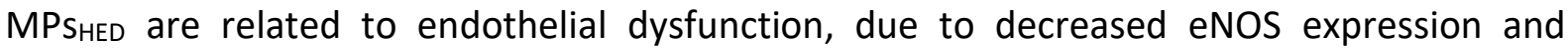
increased ROS release.

\subsection{Differential effect of $M \mathrm{MS}_{\mathrm{ND}}$ and $\mathrm{MPS}_{\mathrm{HED}}$ on cav-1, ICAM-1 expression and F-actin organization on HUVECs}

$M P S_{N D}$ and MPSHED can have a differential effect on the expression of some endothelial

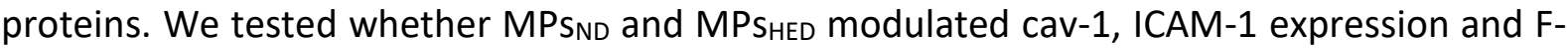
actin organization.

$M_{\text {MD }}$ and MPS $S_{\text {HED }}$ induced a differential effect on vascular cav-1 and ICAM-1 expression (Fig. 3A). Indeed, our results showed a significant difference between the effect of MPSND and that

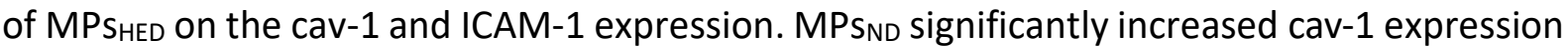
without any effect on ICAM-1 expression. Otherwise, MPSHED significantly decreased cav-1 expression up to $\sim 50 \%$ whereas significant increased ICAM-1 expression by about 2-fold approximately (Fig. 3B). Despite of this, both MPSND and MPSHED treatment did not affect HUVECs cytoskeleton organization observed by F-actin (Fig. 3A). These results showed that both MPS $S_{N D}$ and MPSHED significantly affected the expression of some endothelial proteins and that the HED can change the MPs bioactivity related to endothelial protein expression.

\section{Discussion}


The relationship between the hypercaloric diets (high in both fat and carbohydrates) and coronary atherosclerosis has been a subject of some dispute for many years. Data from early investigations suggested that HED consumption constitutes, an important feature of nutrition transition reflected in the national diets of countries and contributing cause of MetSassociated cardiovascular events $[1,2]$.

Dyslipidemia is one of the major components of MetS. It is associated with hyperlipemia and chronic, low-grade, systemic inflammation leading to atherosclerosis in both mice and humans. Among the actors involved in the development of atherosclerosis, it has been shown that MPs promote atherosclerosis by inducing endothelial dysfunction and upregulating release of proinflammatory mediators.

This study showed that MPs released during a HED-induced dyslipidemia had a dual effect on the development of atherosclerosis by an antiatherogenic action by reducing the endothelial expression of cav-1, and a proatherogenic action by increasing the endothelial expression of ICAM-1 and endothelial ROS production. We previously reported that a HED may induce dyslipidemia and modify levels of circulating MP subpopulations mainly monocyte/neutrophil $(\mathrm{CD} 11 \mathrm{~b}+)$ and procoagulant $(\mathrm{AV}+)$ MPs. These were accompanied by endothelial cav-1 $\beta$ overexpression and aortic ROS in situ production in P. obesus [12].

NO produced by eNOS is the key endothelium-derived relaxing factor regulating vascular function. In addition to its involvement in the local regulation of vascular tone, NO is an inhibitor of coagulation, inflammation and oxidative stress [13].

In this study, treatment with either $M \mathrm{~S}_{\mathrm{ND}}$ or MPS $\mathrm{S}_{\mathrm{HED}}$ caused a significant reduction of the maximal of relaxation induced by acetylcholine, which traduced impairment on endothelial function. These results could be explained by the existence of two distinct mechanisms imprinted by MPs released from both groups of $P$. obesus. The effects of MPS $S_{N D}$ might be related to the eMPs level and their ability to affect eNOS activity and consequently to control of vascular tone. Previously, we have demonstrated that ND group had higher eMPs level compared to the HED group [12]. eMPs can induce a decrease of NO production in rat aortic rings. This NO reduced production was associated with inhibition of NO synthase activity through its interaction with caveolin [14]. The mechanism by which MPs affect NO bioavailability and eNOS activity depends on PI3K, ERK1/2 as well as the nuclear factor NF-KB pathways [15]. Another possible explanation might be related to the result of the overexpression of caveolin-1 in HUVECs treated with MPSND. MPs might enhance the 
association of eNOS with caveolin-1 and subsequently decrease its activation [16]. In the physiological case and outside any pathological situation, the MPs play an important role in the regulation of the vascular tone. Indeed, preincubation of ECs with platelet MPs (pMPs) enhanced arachidonic acid-induced contractions in the aorta. pMPs delivered TXA2 to ECs and might represent their cellular source since they increase their TXB2 levels by about 25-fold [17]. On the other hand, the effects of MPSHED might depend on the increase of ROS that they induce. In fact, it has been demonstrated that MPs may modify vascular tone by increasing the production of ROS and subsequently impairing endothelial NO production. Indeed, injection of T-lymphocyte MPs into mice reduced NO production and increased ROS levels which impaired acetylcholine-evoked endothelial relaxation [16]. Thus, NO production could be affected not only by ROS levels but also by eNOS expression. In this study, MPSHED induced decreased eNOS expression in HUVECs. Functionally, MPs from diabetic rat plasma were able to reduce expression of eNOS in target carotids and decrease acetylcholine-induced endothelium-dependent relaxation on carotid artery rings [18]. Therefore, MPs from patients with myocardial infarction decreased flow induced endothelial relaxation and downregulate eNOS expression [19].

Our results did not corroborate with those reported by Agouni et al. study related to the effects the effects of MPs from MetS patients on endothelial dysfunction and vascular reactivity. In fact, Agouni et al. demonstrated that circulating MPs from MetS patients influenced endothelial dysfunction by impairing endothelium-dependent relaxation [6]. These discrepancies could be due to the animal model used in the present study. Thus, first, we did not induce a MetS in our experimental model, but one of its component; dyslipidemia, in a state of normoglycemia. Second, the phenotyping of MPs in the Agouni et al. study was marked by an increase in plasma levels of eMP, unlike our study from which we reported a decrease in their concentration. Moreover, dyslipidemic microenvironment, particularly in a normoglycemia circumstance, contains fewer stimuli that may influence the composition and bioactivity of MPs than MetS microenvironement which can also increase the ability of ECs to generate MPs. Although pMPs represented the predominant sub-population in MetS patients, the major effect of MPs on the vascular wall was related to non-pMPs [6].

Thus, amplification of endothelial inflammation and increased ROS production are associated with detrimental cardiovascular alterations linked to the MetS [20]. In our study, we observed that MPs from dyslipidemic P.obesus induced a significant increase in ICAM-1 expression and 
ROS production without affecting the F-actin cytoskeleton. Indeed, it has been shown that MPs may contain $\mathrm{NAD}(\mathrm{P}) \mathrm{H}$, induced superoxide production and enhanced expression of the cell adhesion proteins in cultured ECs [21]. Compared to MPs isolated from ND-fed rats, those from HFD-fed rats were characterized by the ability to stimulate ROS generation as well as VCAM-1 expression and consequently they gave a pro-inflammatory character to the MPs [22].

In the pathogenesis of atherosclerosis, ICAM-1 expression, as well as other cell adhesion proteins, had an important role in leukocyte adhesion and transmigration through endothelial cell-cell junctions or through the EC body toward the underlying tissue [23]. Leukocyte adhesion to the ECs induced endothelial ICAM-1 clustering. This binding solicits the involvement of various actin-binding proteins such as filamin $B, \alpha$-actinin- 4 , and cortactin that allowed the establishment of a link between ICAM-1 and F-actin cytoskeleton [24].

In this study, circulating MPs from dyslipidemic $P$. obesus were characterized by a high level of CD11b+MPs [12] and can induce endothelial ICAM-1 overexpression, unlike those of healthy $P$. obesus. These two conditions constituted a favorable environment allowing that leukocytes such as neutrophils adhere on the EC surface by binding of the neutrophils Mac1 to endothelial ICAM-1 [25]. In human atherosclerosis, ICAM-1 can be transferred to ECs by MPs released from atherosclerotic plaque leading to the recruitment of inflammatory cells [26]. There is a strong relationship between the degree of metabolic disorder, the ability of leukocytes to adhere to ECs and inflammation. Indeed, enrichment of monocyte/macrophages with unesterified cholesterol leads to highly procoagulant MPs release in MetS-associated cardiovascular complication. Unesterified cholesterol-induced MPs provoked extensive leucocyte adhesion to ECs in vivo, and increased ICAM-1 expression in ECs via nuclear factor-kB activation [27]. Moreover, a number of investigations documented the pro-inflammatory effects of MPs. Exposure of ECs to the pMPs caused the expression of ICAM-1, VCAM-1 and E-selectin cell adhesion proteins accompanied by the release of several proinflammatory cytokines such as IL-6, and IL-8 [28]. Barry et al. showed that pMPs increased ICAM-1 expression by delivering arachidonic acid to ECs in a transcellular manner [29].

Besides the proinflammatory and vasculo-aggressive action of circulating MPs from the dyslipidemic $P$. obesus by inducing ICAM-1 overexpression and increased ROS production, these same MPs had both vasculoprotective and anti-atherogenic action. In this study, we have demonstrated that MPSHED significantly decreased cav-1 expression which may, 
therefore, have a protective effect on the blood vessel and provides protection against the development of several vascular diseases. Indeed, the caveolae represented one of the ways by which the MPs can mediate the pro-inflammatory signaling pathways which lead to EC activation [30]. Moreover, mitigate the effects of MPs on EGFR phosphorylation, activation of NF-KB and upregulation of ICAM-1 expression can be achieved by inhibition of caveolindependent endocytosis [30]. Apolipoprotein E knockout mice (or its abnormalities in humans) is associated with a series of pathological conditions including dyslipidemia and atherosclerosis. In these mice, cav-1-/- deficiency may protect from high fat-diet induced atherosclerosis [31]. Nevertheless, the effect of MPS HED $_{\text {on }}$ cav-1 expression may not be sufficient to protect vessels since MPSHED impaired endothelium-dependent relaxation induced by Ach resulting in endothelial dysfunction.

Despite the novelty of our findings, this study had several important limitations. To investigate whether ND and HED affected MPs bioactivity related to vessel relaxation, we incubated rings of the thoracic aorta deriving from mouse with $\mathrm{MP}_{\mathrm{ND}}$ or $\mathrm{MP}_{\mathrm{HED}}$ that are from P.obesus. Also, the effects of MPs from P.obesus were tested on human cultured endothelial cells. The use of MPs from one species (P.obesus) and testing their effects on vessels (mice) or cells (human) of another species represented a real problem in the interpretation of vascular reactivity and cell culture results. This was due to the difficulty of procuring P.obesus. Indeed, the difficulty in breeding and maintaining sand rats in captivity strongly limits their use by researcher despite the unique species that mimics the same development of complications related to diabetes type 2 as in humans.

Further experiments are needed to determine the respective role of each subtype of extracellular vesicles in the endothelial protein expression. Nevertheless, the present study described the relationship between dyslipidemia, and their potential actions via MPs on vascular homeostasis in P. obesus. Our result indicated the dual function MPs in regulating the expression of some endothelial proteins and considering MPs as vectors of key information in vascular cells. However, this study adds new facets to our understanding of the complexity of biological message carried by MPs. These findings suggested that more research must be done to decipher the regulating element which can deviate the actions of the MPs towards a vasculoprotective or vasculoaggressive way. 


\section{Acknowledgments}

The authors declare that they have no conflicts of interest. The authors would like to thank Nassim Bouloudene, Mourad Atir and Dr Manuel Gómez-Guzmán for their technical support. This project was supported by Algerian DAHR (the Development Agency of Health Research), the Ministry of Higher Education and Scientific Research of Algeria, the University of Angers (France) and INSERM (France). The funders had no role in the study design, data collection and analysis, in the decision to publish, or in the preparation of the manuscript.

\section{References}

[1] Asha Devi S, Jyothi B. Dyslipidemia in Metabolic Syndrome: an Overview of Lipoprotein-Related Disorders. International Journal of Cardiology and Lipidology Research 2017;4,6-15.

[2] Rahman T, Hamzan NS, Mokhsin A, Rahmat R, Othman Ibrahim Z, Razali R, Thevarajah $\mathrm{M}$, and Nawawi $\mathrm{H}$. Enhanced status of inflammation and endothelial activation in 
subjects with familial hypercholesterolaemia and their related unaffected family members: a case control study. Lipids Health Dis 2017;16-81.

[3] Anthony JV, Anand MI, Ulrich Siebenlist, and Bysani Chandrasekar. OxLDL induces endothelial dysfunction and death via TRAF3IP2. Inhibition by HDL3 and AMPK activators. Free Radic Biol Med 2014; 70: 117-128.

[4] Zhang H, Zhao Z, Pang X, Yang J, Yu H, Zhang Y, Zhou H, Zhao J. Genistein Protects Against Ox-LDL-Induced Inflammation Through MicroRNA-155/SOCS1-Mediated Repression of NF-kB Signaling Pathway in HUVECs. Inflammation 2017;40(4):1450-1459.

[5] Lara-Guzmán OJ, Gil-Izquierdo Á, Medina S, Osorio E, Álvarez-Quintero R, Zuluaga N, Oger C, Galano JM, Durand T, Muñoz-Durango K. Oxidized LDL triggers changes in oxidative stress and inflammatory biomarkers in human macrophages. Redox Biol 2018;15:1-11.

[6] Agouni A, Lagrue-Lak-Hal AH, Ducluzeau H, Mostefai HA, Draunet-Busson C, Leftheriotis G, and al. Endothelial dysfunction caused by circulating microparticles from patients with metabolic syndrome. Am J Pathol 2008;173(4):1210-1219.

[7] Agouni A, Ducluzeau P, Benameur T. Faure S, Sladkova M, Duluc L, et al. Microparticles from patients with metabolic syndrome induce vascular hyporeactivity via Fas/Fas-ligand pathway in mice. PLoS ONE 2011;6 (11):e27809.

[8] Martinez MC, Andriantsitohaina R. Extracellular vesicles and metabolic syndrome. Circ Res 2017;120:1674-1686.

[9] Mause SF, Weber C. Microparticles, Protagonists of a Novel Communication Network for Intercellular Information Exchange. Circ Res 2010;107(9):1047-57.

[10] Del Conde I, Shrimpton CN, Thiagarajan P, Lopez JA. Tissue-factorbearing microvesicles arise from lipid rafts and fuse with activated platelets to initiate coagulation. Blood 2005; 106:1604-1611.

[11] Diamant M, Tushuizen ME, Sturk A, Nieuwland R. Cellular microparticles: new players in the field of vascular disease? Eur J Clin Invest 2004;34:392-401.

[12] Ousmaal MF, Martínez MC, Andriantsitohaina R, Chabane K, Gaceb A, Mameri S, Giaimis J, Baz A. Increased monocyte/neutrophil and pro-coagulant microparticle levels and overexpression of aortic endothelial caveolin-1ß in dyslipidemic sand rat, Psammomys obesus. J Diabetes Complications 2016;30(1):21-9.

[13] Koppenol WH, Moreno JJ, Pryor WA, et al. Peroxynitrite, a cloaked oxidant formed by nitric oxide and superoxide. Chem Res Toxicol 1992;5:834-842. 
[14] Mahmoud AM, Wilkinson FL, McCarthy EM, Moreno-Martinez D, Langford-Smith A, Romero M, Duarte J, Alexander MY. Endothelial microparticles prevent lipid-induced endothelial damage via Akt/eNOS signaling and reduced oxidative stress. FASEB J 2017;31(10):4636-4648.

[15] Mostefai HA, Agouni A, Carusio N, Mastronardi ML, Heymes C, Henrion D, et al. Phosphatidylinositol 3-kinase and xanthine oxidase regulate nitric oxide and reactive oxygen species productions by apoptotic lymphocyte microparticles in endothelial cells. J Immunol 2008a;180:5028-35.

[16] Martin S, Tesse A, Hugel B, Martínez MC, Morel O, Freyssinet JM, Andriantsitohaina R. Shed membrane particles from $T$ lymphocytes impair endothelial function and regulate endothelial protein expression. Circulation 2004; 109:1653-1659.

[17] Pfister SL. Role of platelet microparticles in the production of thromboxane by rabbit pulmonary artery. Hypertension 2004; 43: 428-433.

[18] Ishida K, Taguchi K, Hida M, Watanabe S, Kawano K, Matsumoto T, et al. Circulating microparticles from diabetic rats impair endothelial function and regulate endothelial protein expression. Acta Physiol 2016; 216 211-220.

[19] Boulanger CM, Scoazec A, Ebrahimian T, Henry P, Mathieu E, Tedgui A, Mallat Z. Circulating microparticles from patients with myocardial infarction cause endothelial dysfunction. Circulation 2001;104: 2649-2652.

[20] Elnakish MT, Hassanain HH, Janssen PM, Angelos MG, Khan M. Emerging role of oxidative stress in metabolic syndrome and cardiovascular diseases: important role of Rac/NADPH oxidase. J Pathol 2013;231(3):290-300.

[21] Burger D, Montezano AC, Nishigaki N, He Y, Carter A, Touyz RM. Endothelial microparticle formation by angiotensin II is mediated via Ang II receptor type I/NADPH oxidase/ RHO kinase pathways targeted to lipid rafts. Arterioscler Thromb Vasc Biol 2011;31:1898-907.

[22] Heinrich LF, Andersen DK, Cleasby ME, Lawson C. Long-term high fat feeding of rats results in increased numbers of circulating microvesicles with pro-inflammatory effects on endothelial cells. Br J Nutr 2015;113(11):1704-1711.

[23] Johnson-Leger C, Aurrand-Lions M, Imhof BA. The parting of the endothelium: miracle, or simply a junctional affair? J. Cell Sci 2000;113:921-933. 
[24] Schnoor M. Endothelial actin-binding proteins and actin dynamics in leukocyte transendothelial migration. J Immunol 2015;194:3535-3541.

[25] Nourshargh S, Alon R. Leukocyte migration into inflamed tissues. Immunity 2014;41:694-07.

[26] Rautou PE, Leroyer AS, Ramkhelawon B, Devue C, Duflaut D, Vion AC, Nalbone G, Castier $\mathrm{Y}$, Leseche G, Lehoux S, Tedgui A, Boulanger CM. Microparticles from human atherosclerotic plaques promote endothelial ICAM-1-dependent monocyte adhesion and transendothelial migration. Circ Res 2011; 108(3):335-43.

[27] Liu ML, Scalia R, Mehta JL, Williams KJ. Cholesterol-induced membrane microvesicles as novel carriers of damage-associated molecular patterns: mechanisms of formation, action, and detoxification. Arterioscler Thromb Vasc Biol 2012;32:2113-2121.

[28] Nomura S, Tandon NN, Nakamura T, Cone J, Fukuhara S, Kambayashi J. High-shearstressinduced activation of platelets and microparticles enhances expression of cell adhesion m1olecules in THP-1 and endothelial cells. Atherosclerosis 2001;158:277-87.

[29] Barry OP, Pratico D, Lawson JA, FitzGerald GA. Transcellular activation of platelets and endothelial cells by bioactive lipids in platelet microparticles. J Clin Invest 1997;99:211827.

[30] Andrews AM, Rizzo V. Microparticle-Induced Activation of the Vascular Endothelium Requires Caveolin-1/Caveolae. PLoS One 2016;11(2):e0149272.

[31] Engel D, Beckers L, Wijnands E, Seijkens T, Lievens D, Drechsler M, et al. Caveolin1 deficiency decreases atherosclerosis by hampering leukocyte influx into the arterial wall and generating a regulatory T-cell response. FASEB J 2011;25(11):3838-48.

Table 1. Blood chemistry, fat weight and body weight gain in P. obesus after 12 weeks of feeding.

\begin{tabular}{|c|c|c|c|c|c|}
\hline Parameter & Units & ND $(n=6)$ & HED $(n=6)$ & & $P$ value \\
\hline Total cholesterol & $\mathrm{g} / \mathrm{L}$ & $0.96 \pm 0.05$ & $1.65 \pm 0.13$ & & $0.0003 * * *$ \\
\hline LDL-C & $\mathrm{g} / \mathrm{L}$ & $0.30 \pm 0.06$ & $0.62 \pm 0.06$ & & $0.0093 * *$ \\
\hline HDL-C & $\mathrm{g} / \mathrm{L}$ & $1.24 \pm 0.52$ & $0.81 \pm 0.11$ & & $0.2648 \mathrm{NS}$ \\
\hline TG & $\mathrm{g} / \mathrm{L})$ & $0.92 \pm 0.12$ & $3.66 \pm 0.44$ & & $0.0011 * *$ \\
\hline Glucose & $\mathrm{g} / \mathrm{L}$ & $0.48 \pm 0.05$ & $0.47 \pm 0.04$ & & $0.2911 \mathrm{NS}$ \\
\hline Insulin & & $65.98 \pm 2.70$ & 4.49 & 0.2606 & NS \\
\hline Subcutaneous fat weight & (g) & $3.93 \pm 0.16$ & $5.87 \pm 0.14$ & & $0.0011 * *$ \\
\hline Visceral fat weight & (g) & $2.75 \pm 0.15$ & $4.17 \pm 0.27$ & & $0.0011 * *$ \\
\hline Body weight gain (4 weeks) & (\%) & $6.31 \pm 1.49$ & $14.58 \pm 1.29$ & & $0.0020 * *$ \\
\hline
\end{tabular}


Values are given as means \pm SEM. Significant differences were analyzed using Mann-Whitney test and indicated by $* \mathrm{P}<0.05,{ }^{* * \mathrm{P}}<0.01,{ }^{* * *} \mathrm{P}<0.001$ compared with ND group. NS $=$ not significant.

\section{Figure legends}

Figure 1: Effect of $\mathrm{MPS}_{\mathrm{ND}}$ and $\mathrm{MPs}_{\mathrm{HED}}$ on ex vivo vessel relaxation induced by $\mathrm{ACh}$

(A) Endothelium-dependent relaxation induced by Ach was evaluated by myography. The relaxation was expressed as a percentage of relaxation level. Treatment with both MPSND and MPSHED induced a significant reduction of the maximal of relaxation induced by Ach compared to untreated vessels. Histograms show the maximal effect of Ach in the absence and in the 
presence of MPs (B). Values are expressed as percentage of relaxation (\%) and the data represent the mean \pm SEM $(n=6) * P<0.05$ vs Ctrl.

\section{Figure 2: Differential effect of $M_{S_{N D}}$ and MPS HED $_{\text {on }}$ eNOS expression and oxidative stress on HUVECs}

(A) Confocal image staining of eNOS (green) and superoxide anion production by DHE (red), in HUVECs.

(B) Histograms show fluorescence intensity of HUVECs eNOS staining and superoxide anion production assessed by Image $J$. In comparison with MPSND, MPSHED significantly decreased eNOS expression and increased ROS production. Data represent arbitrary units (A.U.) of the mean \pm SEM ( $n=$ 6). $* \mathrm{P}<0.05, * * \mathrm{P}<0.01$.

\section{Figure 3: Differential effect of MPSND and MPSHED on cav-1, ICAM-1 expression and F-actin organization on HUVECs}

(A) Confocal image staining of cav-1 (red), ICAM-1 (green) and F-actin (red), in HUVECs. (B) Histograms show fluorescence intensity of HUVECs cav-1 staining (red) and ICAM-1 (green) assessed by Image J. MPS $_{\text {ND }}$ and MPSHED have a differential effect on vascular cav-1 and ICAM-1 expression. MPS

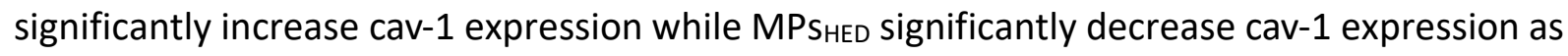
well as a significant increase in ICAM-1 expression. Data represent arbitrary units (A.U.) of the mean \pm SEM $(\mathrm{n}=6) . * \mathrm{P}<0.05, * * \mathrm{P}<0.01, * * * \mathrm{P}<0.001$.

\section{Figure 1}


A

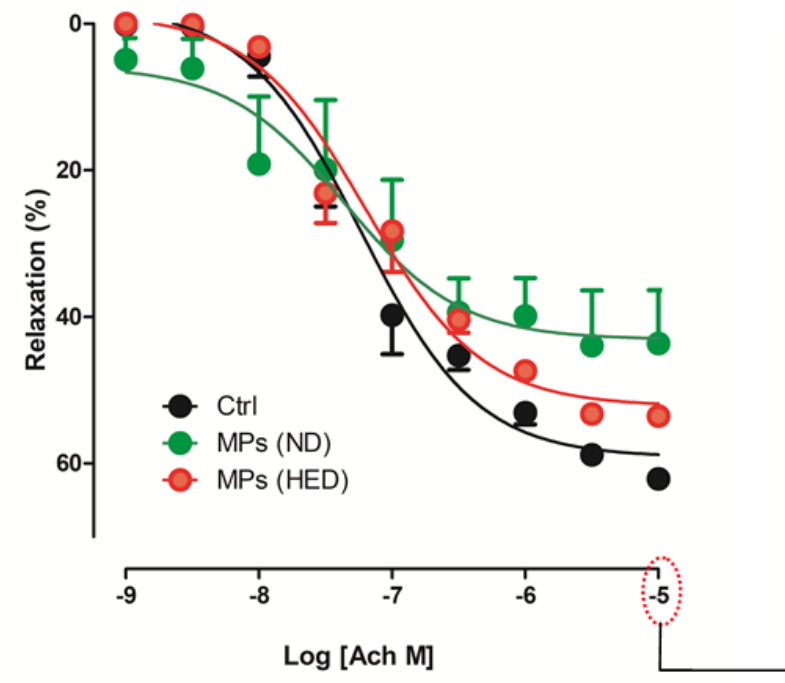

B

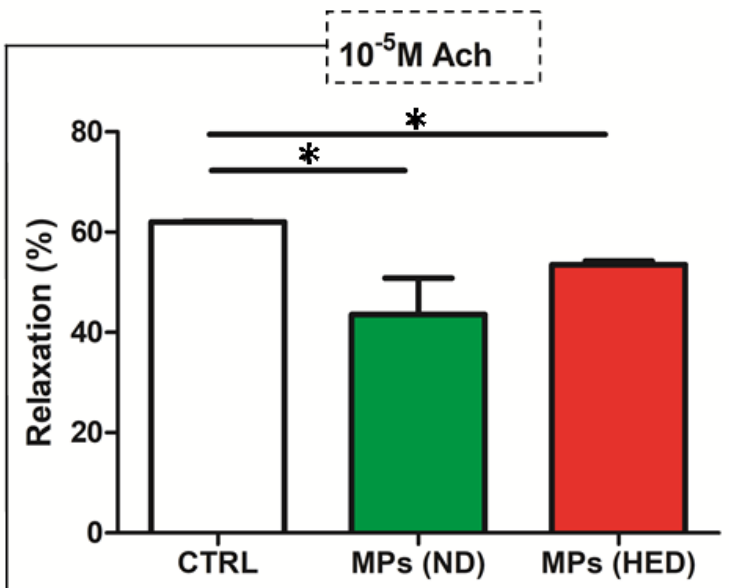

Figure 2

A

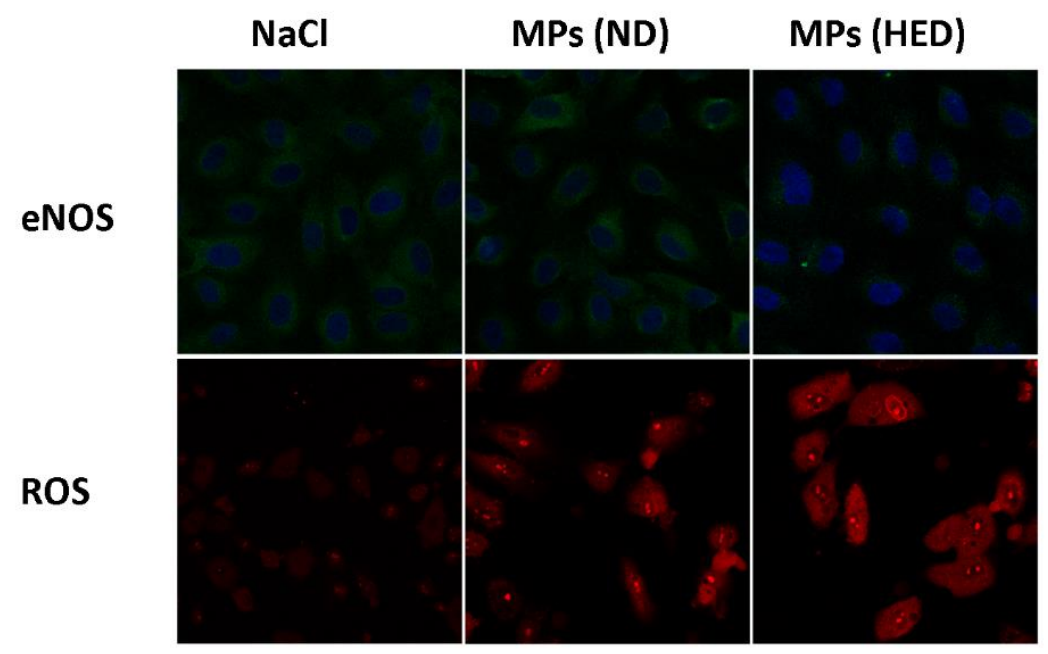

B
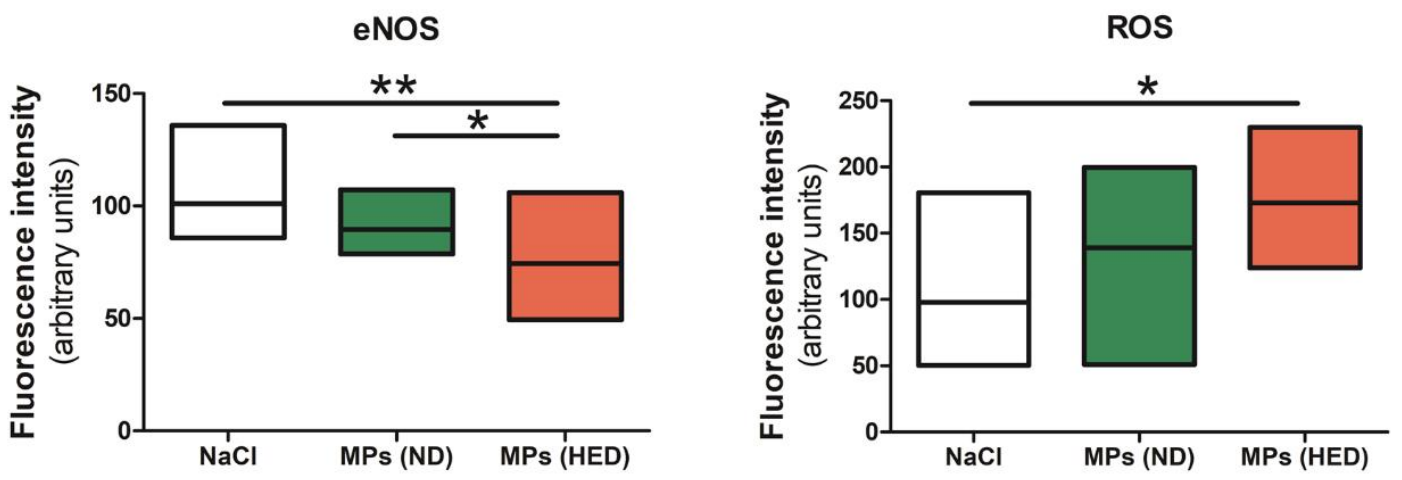

Figure 3 
A

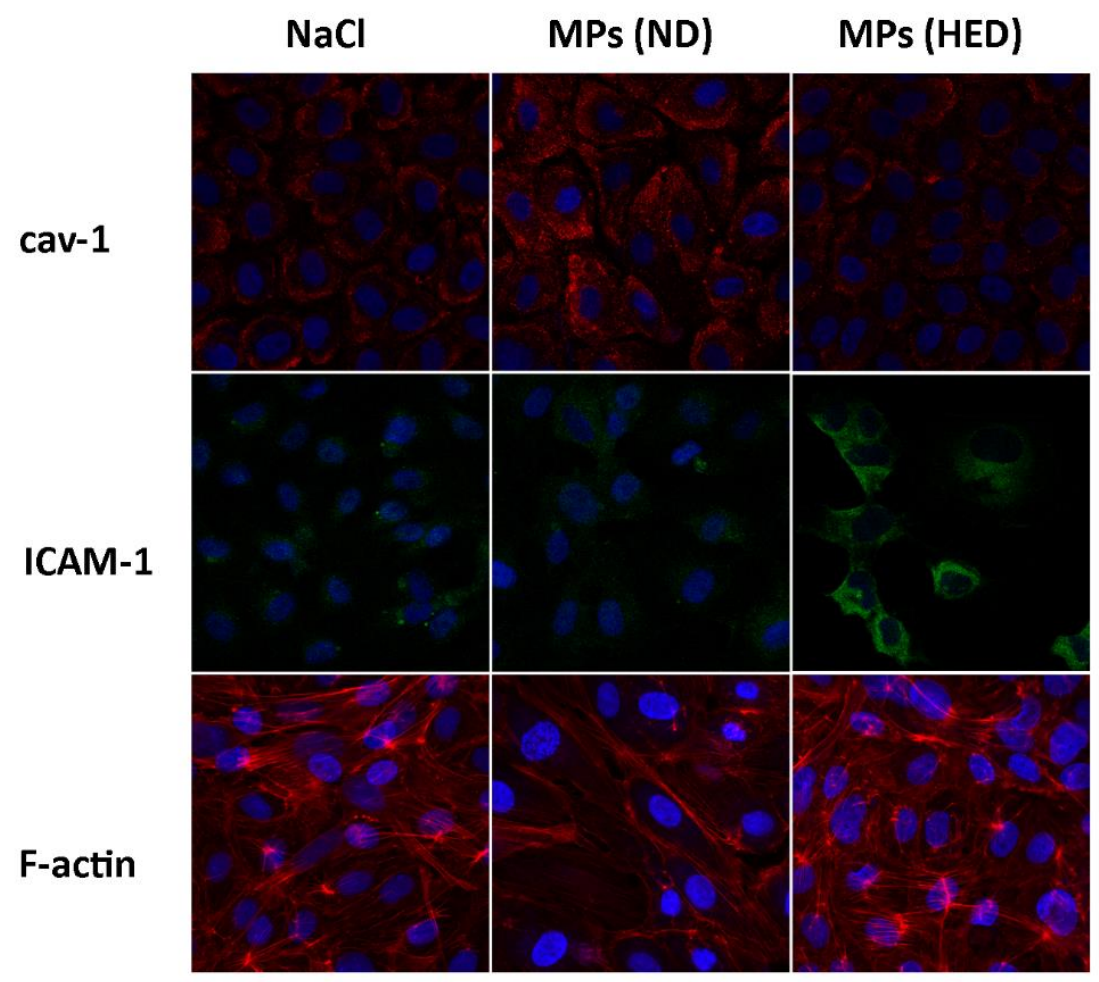

B
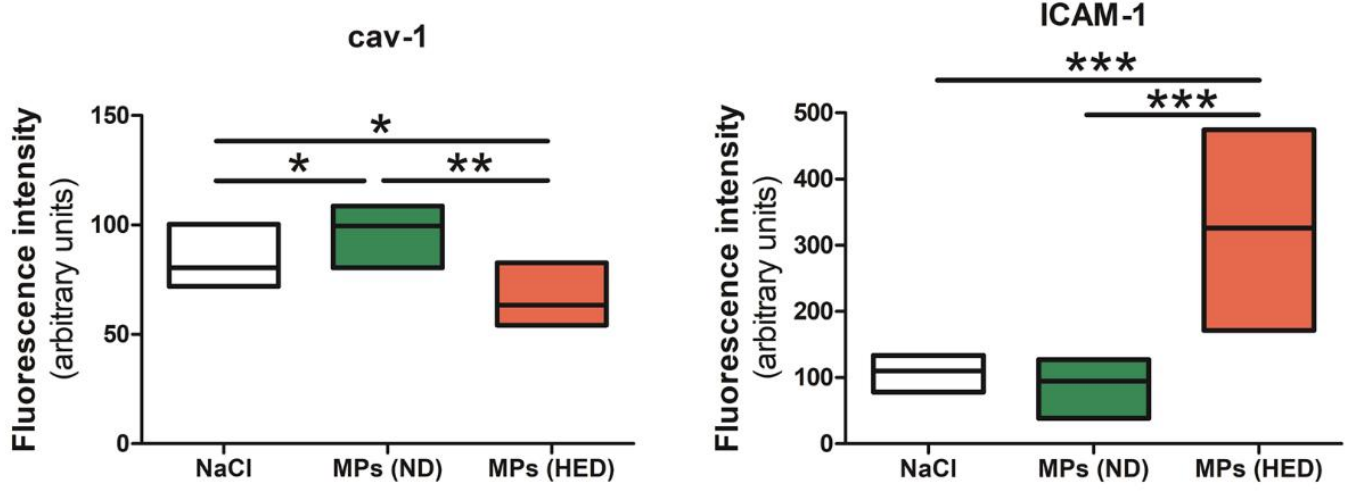

Graphical abstract 


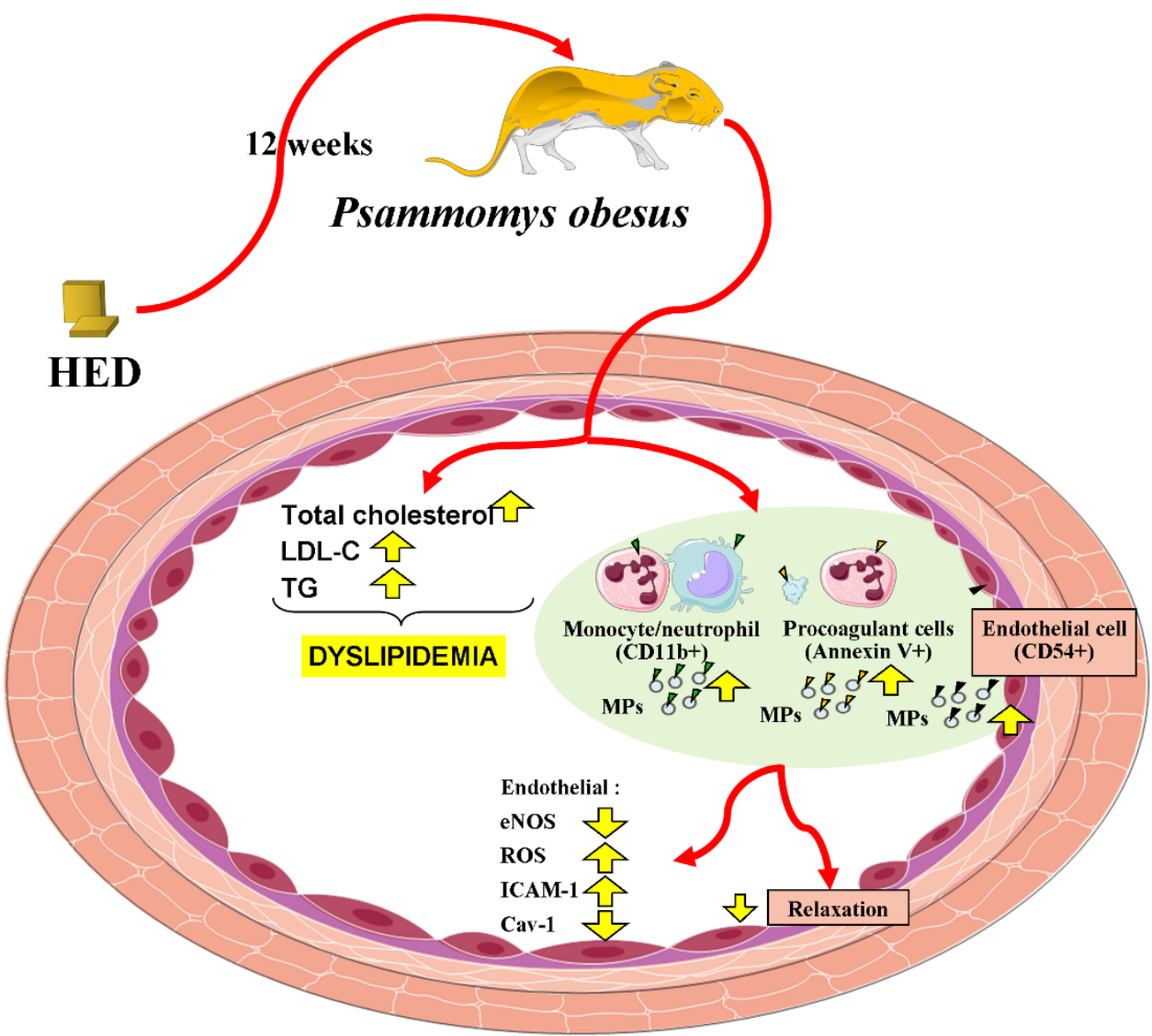

